\title{
The well-known and less well-known benefits of vaccines
}

\author{
Jean-Pierre Michel $^{1}$ D \\ Received: 1 March 2020 / Accepted: 17 June 2020 / Published online: 29 June 2020 \\ (c) Springer Nature Switzerland AG 2020
}

Keywords Vaccines $\cdot$ Global health $\cdot$ Public health $\cdot$ Cardio-vascular prevention $\cdot$ Antimicrobial resistance

\section{Introduction}

With the outbreak of a new coronavirus (COVID-2019) that has been rearing its ugly head for several weeks in China and worldwide [1-3], the international press and the Coalition for Epidemic Preparedness Innovations have already advocated the urgent need to develop an appropriate vaccine [4]. This request illustrates without a doubt that although current uptake of adult vaccines is still too low in Italy, and in Europe in general [5], vaccines have a powerful (if not magic) perceived efficacy for the general public. Indeed, everyone seems to have understood that vaccines train the immune system to recognize and respond to a pathogen by mounting a rapid and effective immune defence, preventing the establishment of infection or disease, or decreasing the disease severity [6].

As stressed in the paper by Antonelli Incalzi and colleagues in this issue [5], vaccine hesitancy is linked to "the constant flow of contradictory, distorted or plainly false news about vaccines in the media, and much of this information stems from sources that are not scientifically robust". Disinformation of the public about vaccination is not compensated for by medical and paramedical personnel, in whom there is a notable lack of training, and who are often not updated about the burden of vaccine preventable diseases (VPDs) [7]. Yet, the fight against VPDs is a major public health priority. The tremendous positive clinical, social and economic impacts of vaccines on preventable infectious diseases are part of a multidimensional and integrated approach to healthy ageing. In their paper, Antonelli Incalzi et al. propose homogeneous and efficacious strategies to scale up vaccination rates from children to seniors in Italy [5], recipes that will be very useful in other European countries. Among

Jean-Pierre Michel

Jean-pierre.michel@unige.ch

1 Medical University of Geneva, Geneva, Switzerland the most important recommendations, the following stand out in particular:

- Set-up of a centralized registry of immunization, enabling continuous monitoring at regional and national levels, which will enable epidemiological evaluation of the impact of vaccinations as well as their socio-economic benefits.

- Implementation of specific teaching about vaccines and vaccination, not only in paediatrics or public health but also in internal and geriatric medicine in all medical and health care faculties.

- Development of a wide-reaching, attractive and repeated media campaign about vaccinations, explaining how they positively impact survival and life expectancy.

Moreover, it is now scientifically proven that vaccines have a significant efficacy on the prevention of cardio- and neuro-vascular events. These benefits are only known to a few professionals [8], who are also aware of the expected benefits of vaccines in the fight against another major health threat, namely antimicrobial resistance.

\section{The well-known and too often forgotten anti infectious efficacy of current vaccines}

As stressed by Antonelli Incalzi et al., vaccination (for adults as well as for children) is recognized as a right and a social value for all. In 2017, thirty-six different vaccines were developed, and over the last thirty years, a number of vaccines have appeared, using firstly live-attenuated strains, then killed whole organisms and more recently, protein or polysaccharide subunits technology [9]. These developments have enabled comparison of morbidity and mortality data on VPDs between the pre-vaccine era and the modern era, across various regions of the world (including Australia, Canada, the UK and the 
USA). These comparisons show an estimated 68 to $100 \%$ reduction in preventable infectious diseases such as congenital rubella, diphtheria, hemophilus influenza $\mathrm{A}$, measles, mumps, pertussis, rubella, tetanus and indeed smallpox [9]. These findings explain the observation that communicable diseases worldwide decreased from $33 \%$ of total deaths in 1990 to $25 \%$ in 2010 [10], thus preventing more than 2.5 million deaths annually from communicable diseases [11]. A related question is whether vaccines have other beneficial impacts on health, in addition to preventing infectious diseases.

\section{Positive vaccine outcomes on the global health burden}

The positive impact of seasonal influenza (flu) vaccination on cardiovascular health was first suspected in the 1990s by Siscovick et al., who observed in a population-based case-control study of 342 cases of primary cardiac arrest (registered from 1988 to 1994 in the Washington area) and 549 demographically similar controls that flu vaccination appeared to be associated with a reduced risk of primary cardiac arrest [odds ratio $(\mathrm{OR})=0.51$ (95\% confidence interval (CI) $0.33-0.79)$ ] [12]. This hypothesis was subsequently confirmed by a randomized, controlled trial including 301 patients hospitalized for myocardial infarction or planned angiography/stenting, showing that mortality at one year was significantly lower in patients who were vaccinated compared to non-vaccinated patients [relative risk of cardiovascular death at 1 year with vaccination $=0.34(95 \%$ CI 0.17-0.71)] [13, 14]. Another randomized, double blind, placebo-controlled study of two population groups (vaccinated vs non-vaccinated) that had 12-month follow-up of 658 optimally treated coronary artery disease patients $(72 \%$ men, mean age $59.9 \pm 10.3$ years) also demonstrated differences in cardiovascular death and coronary revascularization in vaccinated patients [hazard ratio (HR) for ischemic coronary events at 1 year $0.54 ; 95 \%$ CI 0.29-0.99)[15]. These results prompted the American Heart Association and American College of Cardiology to recommend influenza vaccine (inactivated vaccine administered intra-muscularly) for secondary prevention in persons with coronary and other atherosclerotic diseases [16].

More recent evidence has been provided by two successive data analyses from the Taiwan Longitudinal Health Insurance Database (1996-2008). Both concern the prevention of cardiovascular events by flu vaccine. The first study involved 7722 patients aged over 55 years with chronic obstructive pulmonary disease (and without known cardiovascular disease) and showed a significant decrease in the cumulative event rates for acute coronary syndrome in vaccinated versus non-vaccinated individual $(p<0.001)$. Moreover, persons who got four flu vaccines during the course of the study period were better protected against hospitalization for acute coronary syndrome than those who received only one vaccine $(p<0.001)$ [17]. The second analysis from the same database included 4,406 patients aged over 55 years, with chronic kidney disease (without known cardiovascular disease) [18]. Again, patients who received repeated flu vaccines $(N=4)$ had significantly lower cumulative event rates for acute coronary syndrome compared to those who received only one flu vaccine during the study period $(p<0.001)$ [18]. Furthermore, CKD patients receiving influenza vaccination had a lower risk of hospitalization for heart failure [adjusted HR, 0.31 (95\% CI 0.26-0.39), $p<0.001$ ] [18]. Finally, the protective effect of flu vaccination on cardiovascular events was also demonstrated in a Danish nationwide cohort study including all patients over 18 years of age and diagnosed with heart failure (from January 2003 to June 2015) $(n=134,048)$. After the diagnosis of heart failure, one flu vaccination alone was sufficient to significantly reduce cardiovascular mortality $[\mathrm{HR}=0.82(0.81-0.84)]$, while four vaccinations were even more protective $[\mathrm{HR}=0.72$ (0.69-0.74)] $(p<0.001)$ [19].

Another frequent disease among older adults is herpes zoster, and it has been shown that there is an increased risk of stroke after clinically proven onset of herpes zoster in adults between the age of 50 and 60 years[20]. Here again, the positive preventive efficacy of herpes zoster vaccination was recently demonstrated by a cross-sectional nationwide telephone survey of 265,568 non-institutionalized US adults aged 50 to 79 years old [21]. After stratification of participants into six 5-year age groups, Cox proportional hazards analysis indicated that those without zoster vaccination were at significantly higher risk of stroke compared to those receiving vaccination with the live attenuated vaccine $(\mathrm{HR}=1.73,95 \%$ CI 1.71-1.76) [21].

This cumulating body of evidence now clearly testifies to the incontestable beneficial effect of flu vaccination on the onset of cardiovascular events (coronary artery disease, heart failure and cardiovascular death). Moreover, live attenuated herpes zoster vaccine reduces the incidence of postherpes zoster stroke. It is evident that up to now, the positive outcomes of immunization on all cardiovascular events in the older population are most definitely not receiving enough attention [22].

\section{Positive vaccine outcomes on antimicrobial resistance}

In the European Union, approximately 25,000 people die each year from resistant infections, which are estimated to engender an annual financial burden in the order of 1.5 billion Euro [23]. Among the many well-known measures that can control antimicrobial resistance, which include 
sanitation and hygiene, development of rapid diagnostic tests, education to avoid inappropriate antibiotic use, promotion of antibiotic stewardship and elimination of routine antibiotic use in livestock production, an additional avenue towards eradicating resistance is the development of new antimicrobial agents and vaccines [24]. However, the time to first detection of human pathogens resistant to antimicrobial drugs is critically shorter and shorter, while new antibiotics are becoming scarce [25]. In the USA, between 2000 and 2014, the level of antibiotic resistance associated with pneumococcal pneumonia, Staphylococcus aureus, Escherichia coli and Enterobacter spp. reached 34\%, 45\%, 55\% and $88 \%$, respectively [26], even without taking into account the proportion of strains that are resistant to more than one antibiotic class.

On the contrary, vaccines do not acquire significant resistance. This makes them an attractive solution in the fight against antimicrobial resistance, by protecting people against major infectious diseases such as influenza or pneumococcal pneumonia, thereby reducing the spread of disease and the use of antibiotics. Moreover, herd immunity protects not only vaccinated people, but also those who cannot be immunized, such as the immunocompromized. Antipneumococcal vaccines that protect against multiple strains also decrease the density of oral carriage of the microbes and thereby diminish genetic exchanges of resistance, even though a few serotypes that are not contained in the vaccine (A19) have emerged. Two years after the introduction of the 13-valent pneumococcal conjugate vaccine (PCV13) in 2010, the resistance of macrolides, cephalosporins, tetracyclines and penicillin decreased, respectively, by $63 \%, 81 \%$, $81 \%$ and $83 \%$ [24], which is very impressive. Therefore, while currently available vaccines are considered as excellent tools to fight against antimicrobial resistance, it is nonetheless necessary to facilitate access to vaccines, increase coverage rates and accelerate the development and licensing procedures of new vaccines [24]. The WHO has already identified possible targets for new vaccines, and clinical trials will investigate extended epidemiological outcomes, including the impact on clinical prescription of antibiotics and reduction in antimicrobial resistance [22].

The useful public health paper from Antonelli Incalziet al. in this issue [5] underlines with strong arguments and acknowledgeable scientific honesty, the need for increased vaccine awareness among the general public, health care professionals and health care deciders. To succeed in the current era marked by successive new infectious pandemics, vaccine policies must be better harmonized, widely disseminated and constantly updated. The less well-known effects of vaccines on the prevention of cardiovascular events and reduction of antimicrobial resistance are good examples of the constant and growing interest of lifelong immunization.
Funding No financial support.

\section{Compliance with ethical standards}

Conflict of interest No conflict of interest.

Statement of human and animal rights This article does not contain any studies with animals performed by any of the authors.

Informed consent For this type of study, formal consent is not required.

\section{References}

1. Rubin EJ, Baden LR, Morrissey S, Campion EW (2020) Medical journals and the 2019-nCoV outbreak. N Engl J Med. https://doi. org/10.1056/NEJMe2001329

2. Zhu N, Zhang D, Wang W et al (2020) A novel coronavirus from patients with pneumonia in China, 2019. N Engl J Med 382:727733. https://doi.org/10.1056/NEJMoa2001017

3. Chen N, Zhou M, Dong X et al (2020) Epidemiological and clinical characteristics of 99 cases of 2019 novel coronavirus pneumonia in Wuhan, China: a descriptive study. Lancet. https://doi. org/10.1016/S0140-6736(20)30211-7

4. Funfrock P (2020) Novel coronavirus 2019-how to mitigate the impact of new and future epidemics? https://www.prote ogenix.science/scientific-corner/custom-assay/novel-coronaviru s-2019-how-to-mitigate-the-impact-of-the-new-and-future-epide mics/?utm_source $=$ newletter\&utm_medium $=$ mailwizz\&utm campaign=full-text. Accessed 18 Feb 2020 (Proteogenix)

5. Antonelli Incalzi R, Bernabei R, Bonanni P et al (2020) Vaccines in older age: moving from current practice to optimal coverage. A multidisciplinary consensus conference. Aging Clin Exp Res. https://doi.org/10.1007/s40520-020-01622-z

6. Fine PE (1993) Herd immunity: history, theory, practice. Epidemiol Rev 15:265-302. https://doi.org/10.1093/oxfordjournals. epirev.a036121

7. Roberts TE, Harden RM (2015) Coalition: the way forward for medical education. Lancet 385:1479-1480. https://doi. org/10.1016/S0140-6736(15)60685-7

8. Siegrist CA (2005) Vaccinology, a new science at odds with beliefs. Rev Med Suisse 1:3-6

9. Seib K, Pollard AJ, de Wals P et al (2017) Policy making for vaccine use as a driver of vaccine innovation and development in the developed world. Vaccine 35:1380-1389. https://doi. org/10.1016/j.vaccine.2016.10.080

10. Lozano R, Naghavi M, Foreman K et al (2012) Global and regional mortality from 235 causes of death for 20 age groups in 1990 and 2010: a systematic analysis for the Global Burden of Disease Study 2010. Lancet 380:2095-2128. https://doi. org/10.1016/S0140-6736(12)61728-0

11. World Health Organization (2020) Global vaccine action plan 2011-2020. https://www.who.int/immunization/global_vacci ne_action_plan/GVAP_doc_2011_2020/en/. Accessed 15 Feb 2020

12. Siscovick DS, Raghunathan TE, Lin D et al (2000) Influenza vaccination and the risk of primary cardiac arrest. Am J Epidemiol 152:674-677. https://doi.org/10.1093/aje/152.7.674

13. Gurfinkel EP, de la Fuente RL, Mendiz O, Mautner B (2004) Flu vaccination in acute coronary syndromes and planned percutaneous coronary interventions (FLUVACS) study. Eur Heart J 25:25-31. https://doi.org/10.1016/j.ehj.2003.10.018 
14. Gurfinkel EP, de la Fuente RL (2004) Two-year follow-up of the FLU vaccination acute coronary syndromes (FLUVACS) registry. Tex Heart Inst J 31:28-32

15. Ciszewski A, Bilinska ZT, Brydak LB et al (2008) Influenza vaccination in secondary prevention from coronary ischaemic events in coronary artery disease: FLUCAD study. Eur Heart J 29:1350 1358. https://doi.org/10.1093/eurheartj/ehm581

16. Davis MM, Taubert $K$, Benin AL et al (2006) Influenza vaccination as secondary prevention for cardiovascular disease: a science advisory from the American Heart Association/American College of Cardiology. J Am Coll Cardiol 48:1498-1502. https://doi. org/10.1016/j.jacc.2006.09.004

17. Sung L-C, Chen CI, Fang YA et al (2014) Influenza vaccination reduces hospitalization for acute coronary syndrome in elderly patients with chronic obstructive pulmonary disease: a population-based cohort study. Vaccine 32:3843-3849. https:// doi.org/10.1016/j.vaccine.2014.04.064

18. Fang YA, Chen CI, Liu JC, Sung LC (2016) Influenza vaccination reduces hospitalization for heart failure in elderly patients with chronic kidney disease: a population-based cohort study. Acta Cardiol Sin 32:290-298. https://doi.org/10.6515/acs201504241

19. Modin D, Jørgensen ME, Gislason G et al (2019) Influenza vaccine in heart failure. Circulation 139:575-586. https://doi. org/10.1161/CIRCULATIONAHA.118.036788

20. Forbes H, Williamson E, Benjamin L et al (2018) Association of herpesviruses and stroke: systematic review and meta-analysis. PLoS ONE 13:e0206163. https://doi.org/10.1371/journ al.pone. 0206163
21. Klaric JS, Beltran TA, McClenathan BM (2019) An association between herpes Zoster vaccination and stroke reduction among elderly individuals. Mil Med 184(Suppl 1):126-132. https://doi. org/10.1093/milmed/usy343

22. Kingwell K (2018) Vaccines take a shot at antimicrobial resistance. Nat Rev Drug Discov 17:229-231. https://doi.org/10.1038/ nrd.2018.8

23. European Commission AMR: a major European and Global challenge. Factsheet. https://ec.europa.eu/health/amr/antimicrobialresistance_en. Accessed 15 Feb 2020

24. Jansen KU, Anderson AS (2018) The role of vaccines in fighting antimicrobial resistance (AMR). Hum Vaccin Immunother 14:2142-2149. https://doi.org/10.1080/21645515.2018.1476814

25. Kennedy DA, Read AF (2017) Why does drug resistance readily evolve but vaccine resistance does not? Proc Biol Sci 284:20162562. https://doi.org/10.1098/rspb.2016.2562

26. Jansen KU, Knirsch C, Anderson AS (2018) The role of vaccines in preventing bacterial antimicrobial resistance. Nat Med 24:10-19. https://doi.org/10.1038/nm.4465

Publisher's Note Springer Nature remains neutral with regard to jurisdictional claims in published maps and institutional affiliations. 